\title{
双层预紧式六维力传感器及其静态标定"
}

\author{
王志军 姚建涛 吴 遥 叶 冬 赵永生 \\ (燕山大学机械工程学院 秦皇岛 066004)
}

\begin{abstract}
摘要: 针对基于传统 Stewart 结构六维力传感器在解耦、刚度等方面性能的不足, 设计并研制一种双层预紧式六维力传感器, 并对其进行静态标定试验研究。所设计的六维力传感器采用球窝锥头式球面副代替传统球副, 有效地减小了球副的摩擦力矩, 另外测量分支只承受压力, 消除了力在换向时对接触球面的冲击, 提高了整体刚度。基于螺旋理论推导出传感器所施加外力 与测量分支轴向力之间的映射关系, 进而说明预紧式六维力传感器的测量原理。采用最小二乘线性化标定方法对传感器进行 静态解耦和测量误差分析, 推导出传感器的标定矩阵和误差矩阵。研制出双层预紧式六维力传感器样机, 并进行静态标定试 验。经过标定试验, 该传感器各方向最大非线性度误差低于满量程的 $1.17 \%$, 最大耦合误差低于满量程的 $1.82 \%$ 。试验结果 表明双层预紧式六维力传感器具有很高的测量精度, 可用于工业生产中对六维力测量要求较高的场合。
\end{abstract}

关键词: 六维力传感器 预紧式 双层 球面副 静态标定

中图分类号: TP242 TH823

\section{Dual Layers Pre-stressed Six-axis Force Sensor and Its Static Calibration}

\author{
WANG Zhijun YAO Jiantao WU Yao YE Dong ZHAO Yongsheng
}

(College of Mechanical Engineering, Yanshan University, Qinhuangdao 066004)

\begin{abstract}
According to the deficiency of performance of the traditional six-axis force sensor based on Stewart platform structure on decoupling and stiffness, a pre-stressed six-axis force sensor with dual layers is designed and developed, and the static calibration of the sensor is carried out. The proposed six-axis force sensor uses cone-socket type spherical joint instead of the traditional one, which reduces the friction torque of spherical pair effectively and eliminates the commutation impact force for the measuring limbs compressed all the time, so the overall stiffness is improved. Based on screw theory, the mapping of the applied external force and the axial force of measuring limbs of the sensor is derived, and then the measuring principle of the pre-stressed six-axis force sensor is described. Analysis of static decoupling and measurement error of the sensor is conducted by using linear least squares calibration method, and the calibration matrix and error matrix are deduced. The prototype of the dual layers pre-stressed six-axis force sensor was manufactured and the static calibration is performed. The experimental result shows that the maximum nonlinearity error in all the directions of the sensor prototype is less than $1.17 \%$, and the maximum interference error is less than $1.82 \%$, which indicates that the developed six-axis force/torque sensor possesses high measurement accuracy, and can be used for the industrial production which requires higher six-axis force measurement.
\end{abstract}

Key words : Six-axis force sensor Pre-stressed Dual layers Spherical joint Static calibration

\section{0 前言}

六维力传感器以其能够感知外力和力矩的全 部信息而成为非常重要的一类传感器, 在航空航天、

* 国家自然科学基金(50975245)和河北省自然科学基金(E2011203184)资 助项目。20120309 收到初稿, 20121011 收到修改稿
机械加工、汽车制造、智能化机器人等领域具有重 要的应用价值，其应用已涵盖国防科技和民用工业 的各个领域 ${ }^{[1-3]}$ 。因此, 高精度、高灵敏度且能够适 应一定环境的六维力传感器十分急需并具有非常广 润的应用前景。

经典的 Stewart 平台机构上下平台均为六角形, 再由 6 个移动分支两端通过球面副与六角形的各顶 
点联接, 组成 6 自由度的并联机构。该并联机构在 某一位形下, 将 6 个分支中的移动副替换为单维力 传感器, 此时动平台所受的六维外力与 6 个分支单 维力传感器所受力之间存在一一对应的关系。众多 学者采用这一特性, 将 Stewart 并联结构应用到六 维力传感器设计当中 ${ }^{[4-6]}$, 使得传感器具有结构紧 凑、刚度大、承载能力大、无维间耦合和布置灵活 等优点, 成为六维力传感器设计的最佳构型之一。

DWARAKANATH 等 ${ }^{[5]}$ 和 KANG 等 ${ }^{[6]}$ 分别研制了基 于 Stewart 并联结构的六维力传感器, 并对传感器 进行了结构优化设计。王洪光等 ${ }^{[7]}$ 研制了一种大量 程 Stewart 结构六维力/力矩传感器系统, 并采用分 支轮换法辨识了传感器的结构参数。JIN 等 ${ }^{[8-9]}$ 采用 弹性铰链来替代球面副, 并将弹性敏感元件正交布 置, 得到了刚度各向同性的结构, 为 Stewart 结构 六维力传感器和六维控制器的小型化、实用化奠定 了基础。赵铁石等 ${ }^{[10-11]}$ 研制了一种采用柔性转动副 和柔性万向铰链构成的并联 6-UPUR 六维测力平 台, 并进行了静态解耦试验研究。贾振元等 ${ }^{[12-13]}$ 采 用分载原理, 设计并研制了一种利用压电石英作为 力敏元件的新型并联式轴用压电六维大力传感器, 并进行静态和动态标定试验研究。

基于传统 Stewart 并联结构的六维力传感器采 用球面副作为结构连接副, 因此存在着诸如球面副 摩擦力矩大、易产生间隙、迟滞等问题。文献[14-15] 所设计的六维力传感器在 Stewart 并联结构的基础 上, 上下平台之间增加一个预紧分支, 有效地改善 了上述缺点。为了改进预紧式六维力传感器预紧分 支刚度低的不足, 进一步优化传感器结构, 本文研 制了一种新型的双层预紧式六维力传感器。首先介 绍了球窝雉头式球面副的结构特点, 分析了该传感 器的测量原理。采用线性化标定方法对传感器进行 静态解耦和测量误差分析, 推导出传感器的标定矩 阵和误差矩阵。最后研制出双层预紧式六维力传感 器样机及标定系统, 进行了静态标定试验研究。

\section{1 双层预紧式六维力传感器}

\section{1 球窝雉头式球面副}

传统球面副又称球铰, 它是指组成运动副的两 构件能绕一球心作 3 个独立的相对转动的运动副。 球面副是组成复杂机构的一种非常常见而且重要的 一类运动副。但是, 若要消除球面副接触面间的间 隙, 往往需要对球面副进行预紧, 预紧之后球面副 会产生很大的摩擦力矩, 这就影响了传感器的测量 精度。为了降低球面副摩擦力矩, 文献[14]所提出
的整体预紧平台式六维力传感器在 Stewart 平台结 构的基础上采用球窝锥头式球面副代替传统球副, 使得球面副摩擦力矩大为减小, 进而提高了传感器 的测量精度。图 1 中比较了两种球面副的接触面积。 图 1 中点 $O$ 为球面副的球心; 弧线段 $c_{1} c_{2}$ 为接触面 在分支杆平面内的投影; $f$ 表示分支杆可承受力的 方向。由图 1 可看出, 球窝雉头式球面副在保证接 触强度的情况下可减小接触面积, 从而可有效降低 球面副接触面处的摩擦力矩。由于球窝雉头式球面 副使得分支杆只能承受单方向的压力, 这一问题可 在传感器测力之前, 每个分支上施加一定的预紧力, 保证传感器在测量过程中分支始终保持受压来 解决。

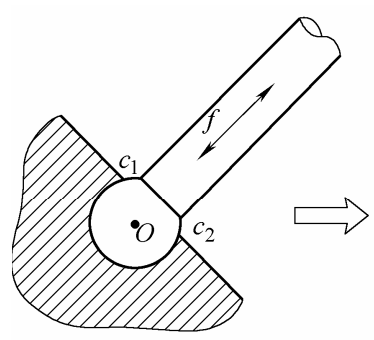

(a) 传统球面副

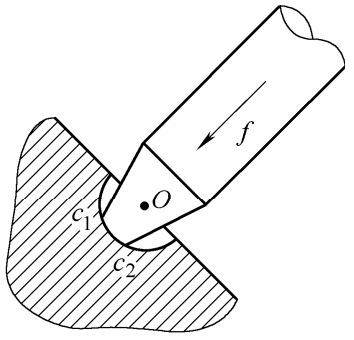

(b) 球窝雉头式球面副
图 1 球面副接触面积对比

球窝雉头式球面副有效地减小了传统球副的 摩擦力矩, 另外使分支杆只承受压力, 从而不存在 过零问题, 消除了力在换向时对接触球面的冲击, 提高了传感器的整体动态刚度。

\section{2 双层预紧式六维力传感器整体结构}

双层预紧式六维力传感器结构如图 2 所示。该 传感器由测力平台、预紧平台、中间平台、支承套 筒、底座、垫片以及 7 个测量分支组成。测量分支 分成两组, 其中 3 个呈圆周对称分布于中间平台上 侧, 另外 4 个半对称分布于中间平台下侧。每个测 量分支由一体式单维力传感器组成, 并与各平台之 间通过单向约束的球窝雉头式球面副连接。由于测 量分支只能承受压力, 若要保证测量分支在测量过 程中始终保持受压, 必须在测量之前对传感器施加 一定的预紧力, 该预紧力大小可由传感器的量程确 定。图 2 中, 支承套筒与底座通过螺栓固连, 6 个 预紧螺栓连接预紧平台与支撑套筒, 压紧各测量分 支从而产生一定的预紧力。预紧平台与支撑套筒之 间为弹性较高的金属材料垫片, 目的是在预紧力施 加后整体连接更加紧密, 结构更加稳定, 并可以通 过改变垫片厚度来实现不同预紧力的施加。

双层预紧式六维力传感器在保留传统 Stewart 结构六维力传感器结构简单、对称性好、无应力耦 合等优点的同时, 还具有以下显著特点: 测量分支 


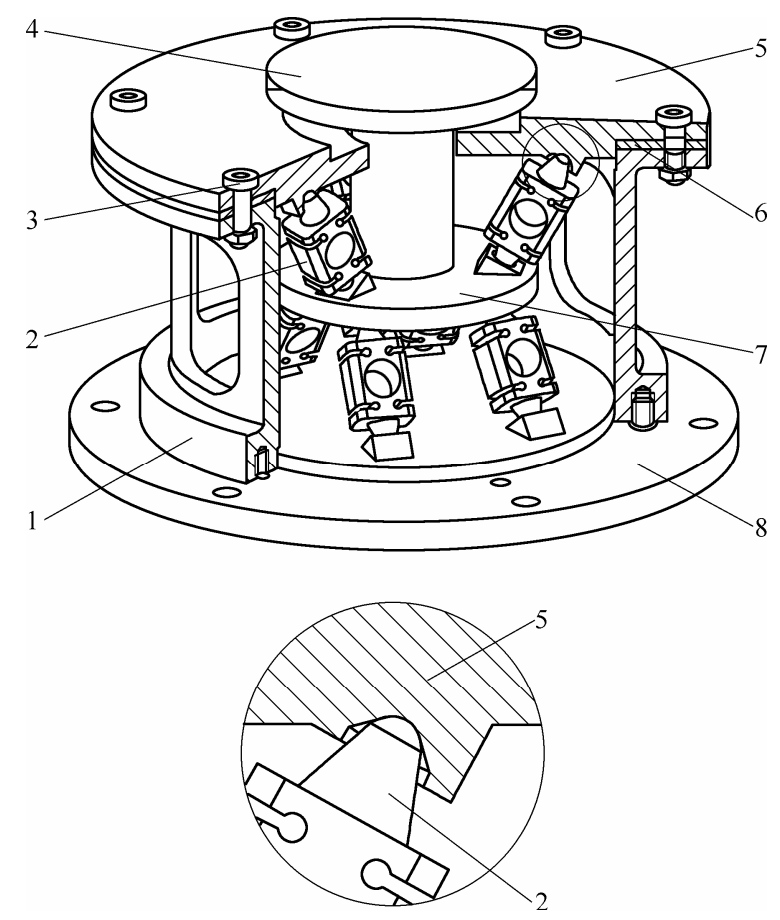

图 2 双层预紧式六维力传感器结构示意图

1. 支承套筒 2. 测量分支 3. 预紧蝶栓 4. 测力平台 5. 预紧平台 6. 垫片 7. 中间平台 8. 底座

不存在过零问题, 因此非线性和迟滞误差将减小; 测量分支分为上下两层, 使结构布置更加灵活, 并 可承受更大的颠覆力矩; 采用整体预紧式方案, 可 有效提高预紧效果, 保证了传感器测量的准确性和 结构的稳定性, 并且增加了传感器的整体刚度。

\section{2 双层预紧式六维力传感器测量原理}

\section{1 传感器数学模型}

并联式六维力传感器依据结构的静力平衡来检 测六维外力, 首先建立传感器的数学模型并推导外 力与测量分支轴向力的映射关系。图 3 为双层预紧 式六维力传感器的结构简图。传感器测量坐标系 $O X Y Z$ 原点建立在中间平台下侧球铰所在平面几何 中心, $X 、 Y$ 轴在中间平台下侧平面内, $Z$ 轴垂直平 面坚直向上。所有球面副分为 4 层分布在中间平台 上侧、下侧、预紧平台、底座上, 各层球面副铰点 均位于同一圆周上。 $\boldsymbol{b}_{1} \sim \boldsymbol{b}_{3} 、 \boldsymbol{b}_{4} \sim \boldsymbol{b}_{7}$ 分别表示中间平 台上下两侧球铰点位置矢量; $\boldsymbol{B}_{1} \sim \boldsymbol{B}_{3} 、 \boldsymbol{B}_{4} \sim \boldsymbol{B}_{7}$ 分别 表示预紧平台球铰点和底座球铰点位置矢量; 并且 有 $\boldsymbol{b}_{1} \sim \boldsymbol{b}_{3} 、 \boldsymbol{B}_{1} \sim \boldsymbol{B}_{3}$ 均匀分布在各自圆周上; $\boldsymbol{b}_{4} \sim \boldsymbol{b}_{7}$ 、 $\boldsymbol{B}_{4} \sim \boldsymbol{B}_{7}$ 关于 $O X Z$ 平面对称分布在各自圆周上。 $R$ 表 示预紧平台和底座球铰点所在圆周半径; $r$ 表示中 间平台上下两侧球铰点所在圆周半径。 $l$ 为测量分 支两端球铰点距离; $H_{1} 、 H_{2}$ 分别为上下两层测量分 支 $Z$ 向距离; $h$ 为中间平台两侧球铰点所在圆周 $Z$
向距离。 $\alpha$ 为球铰点 $\boldsymbol{b}_{4}$ 与 $X$ 轴的夹角; $\beta$ 为球铰点 $\boldsymbol{B}_{4}$ 与其所在圆周圆心连线与平面 $O X Z$ 的夹角。

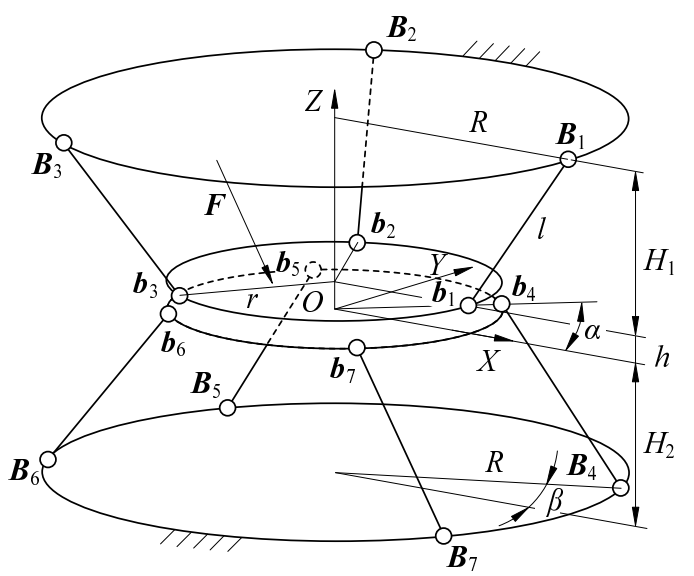

图 3 双层预紧式六维力传感器结构简图

以 $O X Y Z$ 为参考坐标系, 基于螺旋理论建立双 层预紧式六维力传感器所受六维外力与各测量分支 反作用力之间的映射关系为

$$
\boldsymbol{F}=\boldsymbol{G} \boldsymbol{f}
$$

式中, $\boldsymbol{F}=\left(\begin{array}{lllllll}F_{x} & F_{y} & F_{z} & M_{x} & M_{y} & M_{z}\end{array}\right)^{\mathrm{T}}$ 表示作用 在传感器上的六维外力组成的矢量; $\boldsymbol{f}=$ $\left(\begin{array}{lllllll}f_{1} & f_{2} & f_{3} & f_{4} & f_{5} & f_{6} & f_{7}\end{array}\right)^{\mathrm{T}}$ 为测量分支轴向力 组成的矢量; $\boldsymbol{G}$ 为传感器所受六维外力与测量分支 轴向力的映射矩阵, 也称一阶静力影响系数矩阵, 可表示为

$$
\boldsymbol{G}=\left(\begin{array}{cccc}
\frac{\boldsymbol{b}_{1}-\boldsymbol{B}_{1}}{\left|\boldsymbol{b}_{1}-\boldsymbol{B}_{1}\right|} & \frac{\boldsymbol{b}_{2}-\boldsymbol{B}_{2}}{\left|\boldsymbol{b}_{2}-\boldsymbol{B}_{2}\right|} & \cdots & \frac{\boldsymbol{b}_{7}-\boldsymbol{B}_{7}}{\left|\boldsymbol{b}_{7}-\boldsymbol{B}_{7}\right|} \\
\frac{\boldsymbol{B}_{1} \times \boldsymbol{b}_{1}}{\left|\boldsymbol{b}_{1}-\boldsymbol{B}_{1}\right|} & \frac{\boldsymbol{B}_{2} \times \boldsymbol{b}_{2}}{\left|\boldsymbol{b}_{2}-\boldsymbol{B}_{2}\right|} & \cdots & \frac{\boldsymbol{B}_{7} \times \boldsymbol{b}_{7}}{\left|\boldsymbol{b}_{7}-\boldsymbol{B}_{7}\right|}
\end{array}\right)
$$

将传感器结构参数代入到式(2)中, 即可计算出 传感器的一阶静力影响系数矩阵。

\section{2 预紧式六维力传感器静力映射}

式(1)为传感器的静力平衡方程, 解该方程可得

$$
\boldsymbol{f}=\boldsymbol{G}^{-} \boldsymbol{F}+\left(\boldsymbol{I}-\boldsymbol{G}^{-} \boldsymbol{G}\right) \boldsymbol{y}
$$

式中 $\boldsymbol{G}^{-}$一矩阵 $\boldsymbol{G}$ 的广义逆矩阵, 当测量分支刚 度一致时, 可由伪逆矩阵 $\boldsymbol{G}^{+}$确定

$I-7 \times 7$ 维单位矩阵

$\boldsymbol{y}$ 一与 $\boldsymbol{f}$ 相同维数的任一列矢量, 可记为 $\boldsymbol{y}=\left(y_{1}, y_{2}, \cdots, y_{7}\right)^{\mathrm{T}}, \quad \boldsymbol{y} \in \mathbf{R}^{7}$

式(3)中，令

$$
\boldsymbol{f}_{\mathrm{N}}=\boldsymbol{G}^{+} \boldsymbol{F}
$$

式中, $f_{\mathrm{N}}$ 为静力平衡方程的特解, 只与外力有关。

令 $\boldsymbol{\Omega}=\boldsymbol{I}-\boldsymbol{G}^{+} \boldsymbol{G}$, 则有

$$
\boldsymbol{f}_{\mathrm{P}}=\left(\boldsymbol{I}-\boldsymbol{G}^{+} \boldsymbol{G}\right) \boldsymbol{y}=\boldsymbol{\Omega} \boldsymbol{y}
$$

式中, $f_{\mathrm{P}}$ 为静力平衡方程的通解, 并与外力无关, 
可表示传感器的预紧力。

由式(4)、(5), 六维外力空间向七维分支轴向力 空间的线性映射关系可由图 4 表示。图 4 中, 静力 影响系数矩阵 $\boldsymbol{G}$ 为映射矩阵, $\boldsymbol{F}$ 和 $\boldsymbol{f}_{\mathrm{P}}$ 分别为映射 的值域空间和零空间。值域空间 $\boldsymbol{F}$ 为传感器所能承 受的六维外力集合; 分支轴向力空间包含 $\boldsymbol{f}_{\mathrm{N}}$ 和 $\boldsymbol{f}_{\mathrm{P}}$ 两个子空间, 其中零空间 $\boldsymbol{f}_{\mathrm{P}}$ 为不产生任何六维外力 的分支轴向力的集合, 这时的分支轴向力完全由传 感器的机构自身产生。空间 $\boldsymbol{y}$ 为虚拟映射空间, $\boldsymbol{y}$ 中每一组值都有唯一的 $\boldsymbol{f}_{\mathrm{P}}$ 与之相对应。

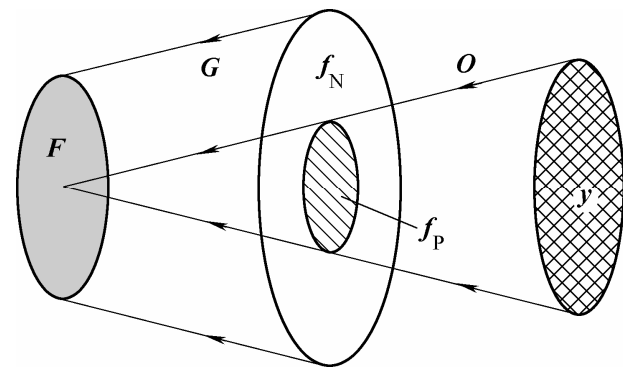

图 4 六维外力与分支轴向力线性映射关系

由图 4 分析可知, 在保证传感器结构非奇异的 情况下, 分支轴向力可以映射到全部的六维外力。 因此, 在传感器检测过程中六维外力施加到测力平 台, 通过中间平台传递到测量分支上, 此时测量分 支反作用力由采集电压得到, 依据外力与分支力之 间的映射关系, 可求得唯一的六维外力值。从而确 保了预紧式六维力传感器测量的唯一性与可行性。

\section{3 传感器的静态标定方法}

由于六维力传感器结构比较复杂, 受设计原理 和制造加工误差等因素的影响, 不可避免地存在测 量误差, 采用标定试验补偿上述测量误差是提高其 测量精度的有效手段。六维力传感器测量误差的评 价形式有各维主方向和耦合方向的平均误差、最大 误差、均方差等。其中各方向最大误差能够更好的 反应传感器实际测量时的误差上限, 因此本文以最 大误差来评价所设计六维力传感器的测量精度。

标定矩阵表达了六维力传感器输入与输出的 线性关系。如果传感器为线性系统, 加载六组线性 无关的力/力矩并采集各分支的输出电压, 即可求得 标定矩阵。但一般情况下, 传感器是一个非线性系 统, 因此在传感器量程范围内采用多点加载, 最小 二乘线性拟合的方法对其进行标定试验。

假设将传感器各方向满量程均分为 $n$ 个加载 点, 所施加的各维标定力组成的矩阵

$$
\boldsymbol{F}_{\mathrm{s}}=\left(\begin{array}{llllll}
\boldsymbol{F}_{x} & \boldsymbol{F}_{y} & \boldsymbol{F}_{z} & \boldsymbol{M}_{x} & \boldsymbol{M}_{y} & \boldsymbol{M}_{z}
\end{array}\right)
$$

$$
\begin{aligned}
& \boldsymbol{F}_{x}=\left(\begin{array}{cccc}
F_{x 1} & F_{x 2} & \cdots & F_{x n} \\
0 & 0 & \cdots & 0 \\
\vdots & \vdots & & \vdots \\
0 & 0 & \cdots & 0
\end{array}\right) \quad \boldsymbol{F}_{y}=\left(\begin{array}{cccc}
0 & 0 & \cdots & 0 \\
F_{y 1} & F_{y 2} & \cdots & F_{y n} \\
\vdots & \vdots & & \vdots \\
0 & 0 & \cdots & 0
\end{array}\right) \\
& \boldsymbol{F}_{z}=\left(\begin{array}{cccc}
0 & 0 & \cdots & 0 \\
0 & 0 & \cdots & 0 \\
F_{z 1} & F_{z 2} & \cdots & F_{z n} \\
\vdots & \vdots & & \vdots
\end{array}\right) \quad \boldsymbol{M}_{x}=\left(\begin{array}{cccc}
\vdots & \vdots & \cdots & \vdots \\
M_{x 1} & M_{x 2} & \cdots & M_{x n} \\
0 & 0 & \cdots & 0 \\
0 & 0 & \cdots & 0
\end{array}\right) \\
& \boldsymbol{M}_{y}=\left(\begin{array}{cccc}
0 & 0 & \cdots & 0 \\
\vdots & \vdots & & \vdots \\
M_{y 1} & M_{y 2} & \cdots & M_{y n} \\
0 & 0 & \cdots & 0
\end{array}\right) \quad M_{z}=\left(\begin{array}{cccc}
0 & 0 & \cdots & 0 \\
\vdots & \vdots & & \vdots \\
0 & 0 & \cdots & 0 \\
M_{z 1} & M_{z 2} & \cdots & M_{z n}
\end{array}\right)
\end{aligned}
$$

式中, $F_{x 1}, F_{x 2}, \cdots, F_{x n}, \cdots, M_{z 1}, M_{z 2}, \cdots, M_{z n}$ 表示全部 的 $6 n$ 个加载力/力矩。

加载 $6 n$ 次后, 可得到测量分支单位力传感器 的输出电压。由式(1), 输出电压与加载力/力矩之间 存在如下关系

$$
\boldsymbol{F}_{\mathrm{s}}=\boldsymbol{G}_{\mathrm{c}} \boldsymbol{U}
$$

式中 $\boldsymbol{F}_{\mathrm{s}}$ 一标定力所组成的矩阵

$\boldsymbol{G}_{\mathrm{c}}$ 一一传感器的标定矩阵, 表达了传感器实 际输入与输出之间的关系

$\boldsymbol{U}$ 一测量分支单维力传感器输出电压所组 成的矩阵

由式(7)可得传感器的标定矩阵

$$
\boldsymbol{G}_{\mathrm{c}}=\boldsymbol{F}_{\mathrm{s}} \boldsymbol{U}^{-}
$$

式中, $\boldsymbol{U}^{-}$为矩阵 $\boldsymbol{U}$ 的逆矩阵, 一般取作伪逆矩阵, 即 $\boldsymbol{U}^{-}=\boldsymbol{U}^{+}=\boldsymbol{U}^{\mathrm{T}}\left(\boldsymbol{U} \boldsymbol{U}^{\mathrm{T}}\right)^{-1}$ 。

标定矩阵得到之后，代入到式(7)中，可得

$$
\boldsymbol{F}_{\mathrm{c}}=\boldsymbol{G}_{\mathrm{c}} \boldsymbol{U}=\boldsymbol{F}_{\mathrm{s}} \boldsymbol{U}^{-} \boldsymbol{U}
$$

式中, $\boldsymbol{F}_{\mathrm{c}}$ 为传感器检测得到的六维力, 可表示为 $\boldsymbol{F}_{\mathrm{c}}=\left(\boldsymbol{F}_{\mathrm{c} x}, \boldsymbol{F}_{\mathrm{c} y}, \boldsymbol{F}_{\mathrm{c} z}, \boldsymbol{M}_{\mathrm{c} x}, \boldsymbol{M}_{\mathrm{c} y}, \boldsymbol{M}_{\mathrm{c} z}\right)$, 其中 $\boldsymbol{F}_{\mathrm{c} x} \sim \boldsymbol{M}_{\mathrm{c} z}$ 均为 $6 \times n$ 维矩阵。

将式(7)、(9)相减并取绝对值, 可得

$$
\begin{gathered}
\Delta \boldsymbol{F}=\left(\begin{array}{llllll}
\Delta \boldsymbol{F}_{x} & \Delta \boldsymbol{F}_{y} & \Delta \boldsymbol{F}_{z} & \Delta \boldsymbol{M}_{x} & \Delta \boldsymbol{M}_{y} & \Delta \boldsymbol{M}_{z}
\end{array}\right)= \\
\\
\left|\boldsymbol{F}_{\mathrm{s}}-\boldsymbol{F}_{\mathrm{c}}\right|
\end{gathered}
$$

式(10)为传感器检测得到的六维力与实际施加 标准值之间的偏差。将式(10)中各方向的最大误差 值与该方向量程之比记为传感器的测量误差, $x$ 方 向的测量误差可表示为

$$
\boldsymbol{E}_{x}=\left(\frac{\max \left(\Delta \boldsymbol{F}_{x 1}\right)}{F_{x \mathrm{~m}}} \frac{\max \left(\Delta \boldsymbol{F}_{x 2}\right)}{F_{y \mathrm{~m}}} \frac{\max \left(\Delta \boldsymbol{F}_{x 3}\right)}{F_{z \mathrm{~m}}}\right.
$$




$$
\left.\frac{\max \left(\Delta \boldsymbol{F}_{x 4}\right)}{M_{x \mathrm{~m}}} \frac{\max \left(\Delta \boldsymbol{F}_{x 5}\right)}{M_{y \mathrm{~m}}} \frac{\max \left(\Delta \boldsymbol{F}_{x 6}\right)}{M_{z \mathrm{~m}}}\right)^{\mathrm{T}}
$$

式中, $\Delta \boldsymbol{F}_{x i}(i=1,2, \cdots, 6)$ 为矩阵 $\Delta \boldsymbol{F}_{x}$ 的第 $i$ 行元素, $\max (\cdot)$ 表示 $(\cdot)$ 中的最大元素。

式(11)中， $\boldsymbol{E}_{x}$ 的第一个元素表示 $x$ 方向力的最 大测量误差, 其余五个元素表示施加 $x$ 方向力时, 其他方向产生的最大耦合误差。

同理, 可得其他方向的最大测量误差和最大耦 合误差, 将其组合成矩阵形式即得到传感器的误差 矩阵

$$
\boldsymbol{E}=\left(\begin{array}{llllll}
\boldsymbol{E}_{x} & \boldsymbol{E}_{y} & \boldsymbol{E}_{z} & \boldsymbol{E}_{\mathrm{m} x} & \boldsymbol{E}_{\mathrm{m} y} & \boldsymbol{E}_{\mathrm{m} z}
\end{array}\right)
$$

式(12)综合反映了六维力传感器的测量误差, 其中对角线元素表示各维主方向的非线性误差, 非 对角线元素表示不同维之间的耦合误差。该误差为 标定试验中各维加载点处的线性拟合值与实际加载 值之间的最大误差, 表达了传感器用于实际检测时 所产生非线性误差的最大值。加载点数目选择不同 所得结果也会不同, 加载点选择越多, 对传感器的 综合测量精度的反应越准确。

\section{4 静态标定试验}

\section{1 双层预紧式六维力传感器样机}

为了验证所提出的球窝雉头式球面副的可行 性与优越性, 研制了双层预紧式六维力传感器样机, 并进行了静态标定试验研究。传感器样机如图 5 所 示, 每个测量分支由一体化标准单维力传感器组成, 用于检测分支轴向力; 其余部分采用超硬铝材料加 工而成, 可以在保证一定刚度的情况下, 有效减小 整体质量。传感器的设计量程: 力 $F_{x \mathrm{~m}}=F_{y \mathrm{~m}}=F_{z \mathrm{~m}}=$ $\pm 100 \mathrm{~N}$, 力矩 $M_{x \mathrm{~m}}=M_{y \mathrm{~m}}=M_{z \mathrm{~m}}= \pm 10 \mathrm{~N} \cdot \mathrm{m}$ 。

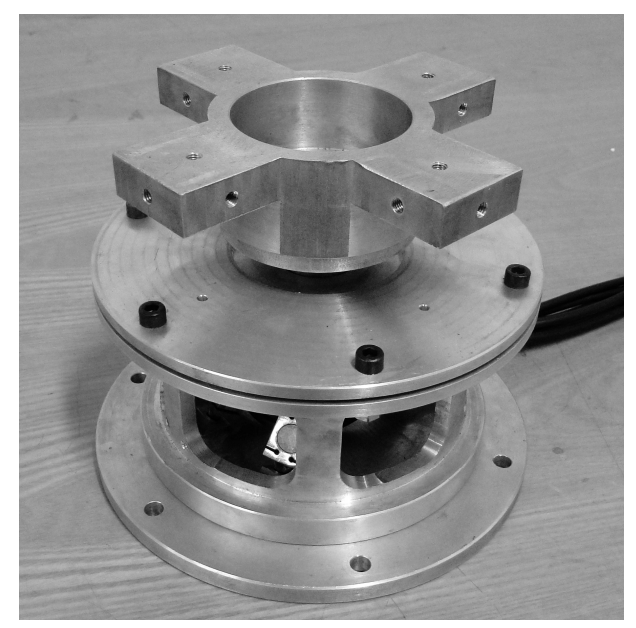

图 5 双层预紧式六维力传感器样机

\section{2 静态标定试验与标定结果}

六维力传感器标定系统主要由加载台、六维力 传感器、砝码、计算机、信号放大和数据采集器以 及直流电源组成。在传感器测力平台上安装有可实 现六维方向力加载的加载块。图 6 表示了各维力与 力矩加载方式, 为了表达清晰, 力矩只表示出了正 方向。标准加载力依靠高精度砝码重力产生, 砝码 通过钢丝绳、导向滑轮与传感器加载块连接。该方 法可以有效减小摩擦力矩、加载力方向等带来的偏 差, 提高了加载力的精度。

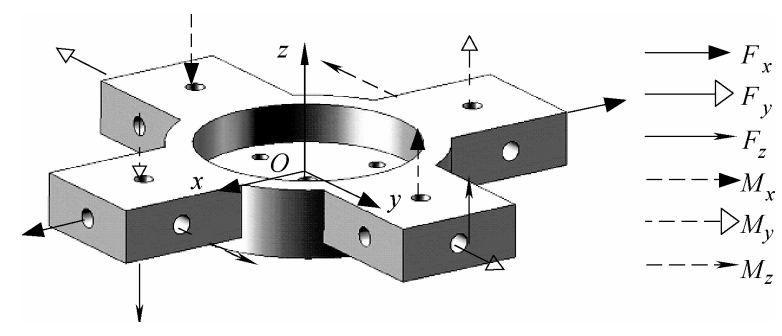

图 6 静态标定六维力施加方式

将传感器的每个方向均分为 20 个加载点进行 逐级加载, 并采集相应的 7 个测量分支输出电压, 由式(8)可得到传感器的静态标定矩阵

$$
\boldsymbol{G}_{\mathrm{c}}=
$$

$\left(\begin{array}{ccccccc}0.021 & -0.010 & -0.009 & 0.023 & -0.022 & -0.027 & 0.025 \\ 0.001 & 0.019 & -0.015 & 0.003 & 0.004 & -0.003 & -0.003 \\ 0.037 & 0.038 & 0.036 & -0.033 & -0.033 & -0.034 & -0.036 \\ 0 & 0.005 & -0.004 & -0.001 & -0.001 & 0.001 & 0.001 \\ -0.005 & 0.003 & 0.002 & -0.003 & 0.003 & 0.003 & -0.003 \\ 0 & 0 & 0 & -0.001 & 0.001 & -0.001 & 0.001\end{array}\right)$

将标定矩阵代入式(9)中, 得到标定之后传感器 对于加载力/力矩的测量结果。图 7 10 表示了加载 $x 、 z$ 方向力和力矩时, 标准加载力值与传感器测量 结果的变化曲线, 可知传感器的输出具有较高的线 性度, 并且维间耦合值较小。

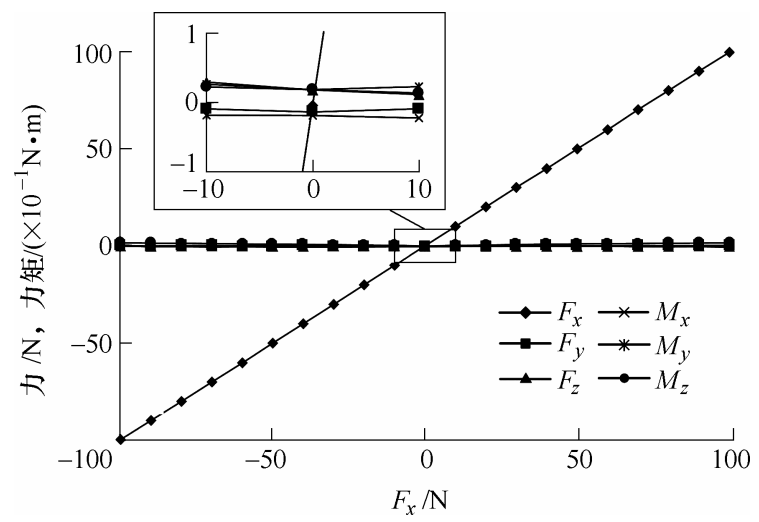

图 7 加载 $x$ 方向力时各维输出值 


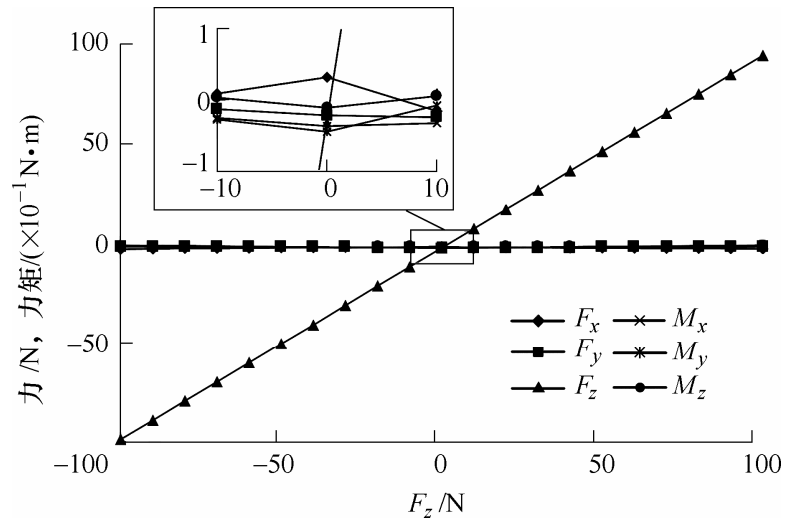

图 8 加载 $z$ 方向力时各维输出值

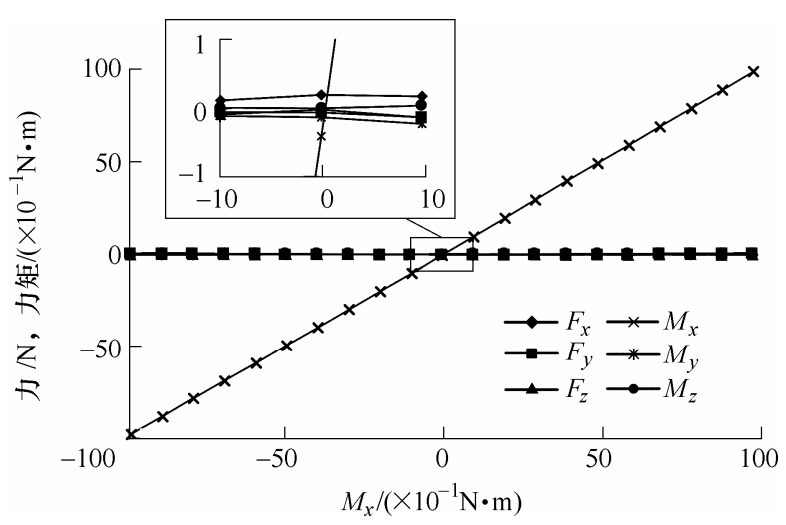

图 9 加载 $x$ 方向力矩时各维输出值

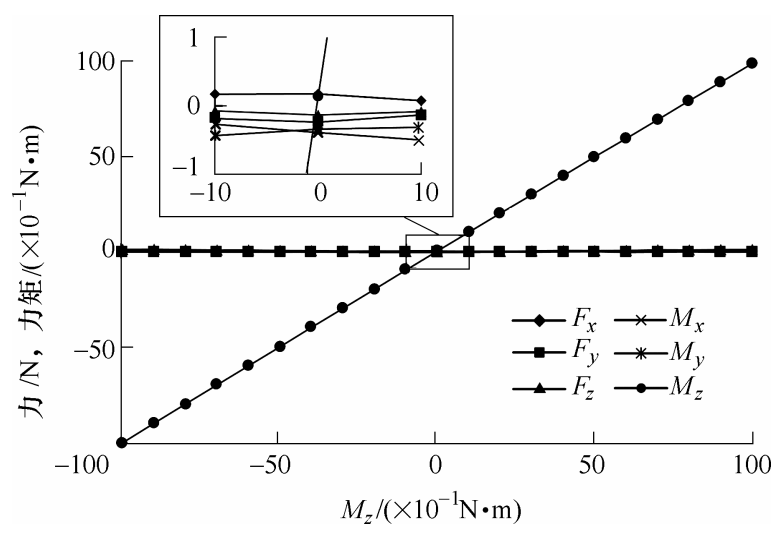

图 10 加载 $z$ 方向力矩时各维输出值

由式(12), 求得传感器的误差矩阵

$$
E=
$$

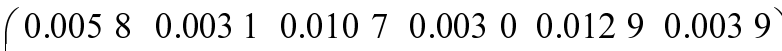
$\begin{array}{llllllllll}0.001 & 0 & 0.005 & 8 & 0.0027 & 0.002 & 6 & 0.007 & 0.005 & 5\end{array}$ $\begin{array}{llllllllll}0.0039 & 0.0044 & 0.005 & 0 & 0.004 & 6 & 0.0059 & 0.0109\end{array}$ $\begin{array}{lllllllll}0.0027 & 0.018 & 2 & 0.010 & 4 & 0.008 & 0.0123 & 0.0061\end{array}$ $\begin{array}{llllllll}0.0065 & 0.0068 & 0.0075 & 0.0084 & 0.0078 & 0.0056\end{array}$ $\left(\begin{array}{lllllll}0.0158 & 0.0019 & 0.006 & 0.0046 & 0.0035 & 0.0117\end{array}\right)$

由误差矩阵可知, 该传感器最大非线性误差为 满量程的 $1.17 \%$; 维间最大耦合误差为满量程的 $1.82 \%$ 。静态标定试验结果表明, 所研制的双层预 紧式六维力传感器具有很高的测量精度, 可用于工
业生产中对六维力测量要求较高的场合。

\section{5 结论}

(1) 研制了一种双层预紧式六维力传感器, 该 传感器具有结构紧凑、耦合低、刚度大、精度高等 优点, 可用于工业生产中对六维力测量要求较高的 场合。

(2) 基于螺旋理论建立了该六维力传感器的输 入外力与输出分支反力之间的映射关系, 并分析了 其进行六维力测量的可行性。

(3) 采用最小二乘的线性化标定方法对传感器 进行静态标定试验研究, 结果表明该传感器的静态 最大测量误差在满量程的 $2 \%$ 以内。

(4) 研究内容对六维力传感器的开发, 特别是 双层预紧式六维力传感器的实用化具有重要的意义。

\section{参 考 文 献}

[1] GAILLET A, REBOULET C. An isostatic six component force and torque sensor[C]// Proceedings of the 13th International Symposium on Industrial Robots, April 17-21, 1983, Chicago, USA. Robotics International of SME, 1983: 102-111.

[2] KIM G S, SHIN H J, YOON J W. Development of 6-axis force/moment sensor for a humanoid robot's intelligent foot[J]. Sensors and Actuators A, 2008, 141: 276-281.

[3] 戈瑜, 吴仲城, 葛运建. 面向应用需求的力/力矩传感 器技术发展动向 [J]. 机器人, 2003, 25(2): 188-192. GE Yu, WU Zhongcheng, GE Yunjian. State of arts and development trends toward application-oriented force/ torque sensors[J]. Robot, 2003， 25(2): 188-192.

[4] 王宣银, 尹瑞多. 基于 Stewart 机构的六维力/力矩传感 器 [J]. 机械工程学报, 2008, 44(12): 118-122.

WANG Xuanyin, YIN Ruiduo, Six-axis force/torque sensor based on Stewart platform[J]. Journal of Mechanical Engineering, 2008, 44(12): 118-122.

[5] DWARAKANATH T A, DASGUPTA B, MRUTHYUNJAYA T S. Design and development of a Stewart platform based force-torque sensor[J]. Mechatronics, 2001, 11(7): 793-809.

[6] KANG C G. Closed-form force sensing of a 6-axis force transducer based on the Stewart platform[J]. Sensors and Actuators A, 2001, 90(1): 31-37.

[7] 王洪光, 赵明扬, 房立金, 等. 一种 Stewart 结构六维 力/力矩传感器参数辨识研究 [J]. 机器人, 2008, 30(6): 548-553. 
WANG Hongguang, ZHAO Mingyang, FANG Lijin, et al. On parameter identification of a Stewart platformbased six-component force/torque sensor[J]. Robot, 2008, 30(6): 548-553.

[8] JIN Zhenlin, GAO Feng, ZHANG Xiaohui. Design and analysis of a novel isotropic six-component force/torque sensor[J]. Sensors and Actuators A, 2003, 109(1): 17-20.

[9] 金振林, 高峰, 赵现朝. 并联结构六维控制器的标定 试验与分析[J]. 机械工程学报, 2005, 41(8): 103-107. JIN Zhenlin, GAO Feng, ZHAO Xianchao. Calibration experiment and analysis for a 6-DOF controller with parallel structure[J]. Chinese Journal of Mechanical Engineering, 2005, 41(8): 103-107.

[10] 赵铁石. 并联 6-UPUR 六维测力平台: 中国, 200610012602[P]. 2006-09-06.

ZHAO Tieshi. 6-UPUR parallel six-axis force measurement platform : China, 200610012602[P]. 2006-09-06.

[11] 石中盘, 赵铁石, 厉敏, 等. 大量程柔性铰六维力传 感器静态解耦的研究 [J]. 仪器仪表学报, 2012, 33(5): 1062-1069.

SHI Zhongpan, ZHAO Tieshi, LI Min, et al. Research on static decoupling of large range flexible joint six-axis force sensor[J]. Chinese Journal of Scientific Instrument, 2012, 33(5): 1062-1069.
[12] 贾振元, 李映君, 张军, 等. 并联式轴用压电六维力/ 力矩传感器 $[\mathrm{J}]$. 机械工程学报, 2010, 46(11): 62-68. JIA Zhenyuan, LI Yingjun, ZHANG Jun, et al. Axial piezoelectric 6-component force/torque sensor based on parallel structure[J]. Journal of Mechanical Engineering, 2010, 46(11): 62-68.

[13] JIA Zhenyuan, LIN Sheng, LIU Wei. Measurement method of six-axis load sharing based on the Stewart platform[J]. Measurement, 2010, 43: 329-335.

[14] 赵永生. 整体预紧平台式六维力传感器: 中国, 99102526.1[P]. 2000-08-16.

ZHAO Yongsheng. Prestressed platform-based six-axis force sensor: China, 99102526.1[P]. 2000-08-16.

[15] HOU Yulei, YAO Jiantao, ZENG Daxing, et al. Development and calibration of a hyperstatic sixcomponent force/torque sensor[J]. Chinese Journal of Mechanical Engineering, 2009, 22(4): 505-513.

作者简介: 王志军, 男, 1983 年出生, 博士研究生。主要研究方向为并 联六维力传感器技术、机器人力控制技术。

E-mail: zjwang@ysu.edu.cn

赵永生(通信作者), 男, 1962 年出生, 教授, 博士研究生导师。主要研 究方向为机器人、传感器、数控加工等理论和技术。

E-mail: yszhao@ysu.edu.cn 